\title{
Entanglement bases and general structures of orthogonal complete bases
}

\author{
Zai-Zhe Zhong \\ Department of Physics, Liaoning Normal University, Dalian 116029, Liaoning, \\ China. E-mail: zhongzaizheh@hotmail.com
}

\begin{abstract}
In quantum mechanics and quantum information, to establish the orthogonal bases is a useful means. The existence of unextendible product bases impels us to study the 'entanglement bases' problems. In this paper, the concepts of entanglement bases and exact-entanglement bases are defined, and a theorem about exact-entanglement bases is given. We discuss the general structures of the orthogonal complete bases. Two examples of applications are given. At last, we discuss the problem of transformation of the general structure forms.

PACC numbers: 03.67.Mn, 03.65.Ud, 03.67.Hk.
\end{abstract}

In quantum mechanics and quantum information, the concept of bases of a Hilbert space is of paramount importance. A product basis (PB) of a Hilbert space of multipartite quantum states is a set of orthogonal product pure-states. In common cases, we always use the standard bases, which are the simplest PBs. However, especially in quantum information theory, one need to use the bases consisting of some entangled pure-states. For instance, in the study of teleportation the Bell's bases, which are maximally entangled states, are very important. Thus, it is necessary that in quantum information we consider various possible orthogonal bases. In principle, a general orthogonal complete basis consists of some product pure-states and some entangled pure-states. Related this, recently a problem discussed in depth is the so-called 'unextendible product basis (UPB)'[1], which must relate to the bases consisting of some entangled pure-states. There are many works about UPB, mainly see Bennett, DiVincenzo, Terhal et al.[1-5], in addition, [6-12], these works show that the UPBs have many quite special properties.

An UPB of a Hilbert space $H$ of multipartite quantum states is a PB $S, S$ spans a subspace $H_{S}$ in $H$, and the complementary subspace $H-H_{S}$ contains no product state. The existence of UPBs means that the structures of general orthogonal complete bases are more complex. Since either a pure-state is a product state, or an entangled state (it may be partial separable), to discuss the structures of general orthogonal complete bases of $H$ is just to discuss how the product pure-states and entangled pure-states assort in the bases. In this paper, first we define the concepts of entanglement basis and exact-entanglement basis, and give a theorem about the exact-entanglement bases. Two examples of applications are given. We discuss the problem of the general structures of the orthogonal complete bases. At last, we consider the transformation problem.

Generally, for a given pure-state we can more easily decide whether it is separable (i.e. a product state), or is entangled. For instance, according to the entanglement of formation[13], only the rank of the coefficient matrix of a bipartite qubit pure-states can decide whether this state is separable, or not. As for a given multipartite qubit purestate, we can use other criteria, e.g. the criteria given by Dür[14] and Kauffman et al.[15], etc.. Since the purpose in this paper is not to study the problem of criteria of separability, for the sake of simplicity, in the following we assume that for any given multipartite pure-state we always can decide whether it is a product state, or is an entangled state.

Consider a multipartite quantum system $H=\otimes_{i=1}^{M} H_{i}$ with $M$ parties of respective dimension $d_{i}$, the total dimensionality of $H$ is $N=\prod_{i=1}^{M} d_{i}$. If an orthogonal complete basis $B=\left\{\left|\omega_{0}>,\right| \omega_{1}>, \cdots, \mid \omega_{N-1}>\right\}$ of $H$ is given, then we always have

$$
\sum_{s=0}^{N-1}\left|\omega_{s}><\omega_{s}\right|=I
$$

where $I$ is the $N \times N$ unit matrix. $B$ can be split into two parts as $S=\left\{\left|\psi_{0}>, \cdots,\right| \psi_{m-1}>\right\}$ and $T=\left\{\left|\varphi_{0}>, \cdots,\right| \varphi_{n-1}>\right\}$, i.e. $\left|\psi_{i}>=\right| \omega_{i}>,\left|\varphi_{j}>=\right| \omega_{j+m-1}>(m+n=N)$, then $B=S \cup T=$ $\left\{\left|\psi_{0}>, \cdots,\right| \psi_{m-1}>,\left|\varphi_{0}>, \cdots,\right| \varphi_{n-1}>\right\}$. Let $H_{S}$ and $H_{T}$, respectively, be the subspaces spanned by $S$ and $T$. For any orthogonal basis $S^{\prime}$ of $H_{S}$, there must be a $m \times m$ unitary matrix $U(m)$ such that $S^{\prime}=S_{U(m)}=\left\{\psi_{0}^{\prime}, \cdots, \psi_{m-1}^{\prime}\right\}$, where $\left|\psi_{i}^{\prime}>=\sum_{s=0}^{m-1}[U(m)]_{i s}\right| \psi_{s}>$. Similarly, any orthogonal basis $T^{\prime}$ of $H_{T}$ can be written as $T^{\prime}=T_{U(n)}=$ $\left\{\varphi_{0}^{\prime}, \cdots, \varphi_{n-1}^{\prime}\right\}$, where $\left|\varphi_{j}^{\prime}>=\sum_{s=0}^{n-1}[U(n)]_{j s}\right| \varphi_{s}>$.

Definition. Consider a multipartite quantum system $H=\otimes_{i=1}^{M} H_{i}$ with $M$ parties of respective dimension $d_{i}$, the total dimensionality of $H$ is $N=\prod_{i=1}^{M} d_{i}$. An entanglement basis $(E B) T=\left\{\left|\varphi_{0}>, \cdots,\right| \varphi_{n-1}>\right\}$ is a set of $n$ entangled pure-states, $\mid \varphi_{j}>(j=0, \cdots, n-1)$, such that an arbitrary linear combination of them steel is an entangled pure- 
state. The subspace $H_{T}$ spanned by an EB $T$ ( $H_{T}$ does not contain any disentangled pure-states) is called an entanglement space (ES). An EBT is called exact-entanglement basis (EEB) if there is a UPB $S=\left\{\left|\psi_{0}>, \cdots,\right| \psi_{m-1}>\right\}$ containing $m=N-n$ product states such that $B=S \cup T=\left\{\left|\psi_{0}>, \cdots,\right| \psi_{m-1}>,\left|\varphi_{0}>, \cdots,\right| \varphi_{n-1}>\right\}$ forms an orthogonal complete basis of $H$. In this case the subspace $H_{T}$ is called an exact-entanglement space (EES), in which all states and the UPB $S$ are orthogonal each other. And we call $B$ a complete basis with an unextendible product basis (CBUPB).

For an EES $H_{E S}$, the corresponding UPB $S$ is in $H_{E S}^{\perp}$, where $H_{E S}^{\perp}$ is the orthogonal complementary subspace of $H_{E S}$. About this, recently Bravy[11] proves a result in the case of tripartite qubit states (the Lemma 3 in [11]). However, it is easily seen that the Lemma 3 and its proof in [11], in fact, can be directly generalized to higher dimensional cases, thus we have the following useful lemma

Lemma 1. For an EES $H_{E S}$, the corresponding UPB in $H_{E S}^{\perp}$ is unique.

As in the above mention, it is necessary that in quantum information we should consider the general orthogonal complete bases. In the first place, we consider the problem what are the conditions of an EB to be an EEB. Another problem is how to describe the general form of orthogonal complete bases. The following theorem can answer the above problems, it,in fact, is a criterion of the existence of EEBs, and it can be used to describe the general structures of orthogonal complete bases.

Theorem 1. The sufficient and necessary conditions for an ES $H_{E S}$ to be an EES are that

(A) The dimensionality $n$ of $H_{E S}$ satisfies $n \leq N-\sum_{i=1}^{M}\left(d_{i}-1\right)-1$.

(B) For any orthogonal basis $T=\left\{\left|\varphi_{0}>, \cdots,\right| \varphi_{N-n-1}>\right\}$ of $H_{E S}$, the uniform mixture $\bar{\rho}=$ $\frac{1}{N-n}\left\{I-\sum_{j=0}^{n-1}\left|\varphi_{j}><\varphi_{j}\right|\right\}$ is separable, where $I$ is the $N \times N$ unit matrix.

Proof. Necessity. Suppose that $H_{E S}$ is a $n$-dimensional EES and $T=\left\{\left|\varphi_{0}>, \cdots,\right| \varphi_{n-1}>\right\}$ is an arbitrary orthogonal basis in $H_{E S}$, then there is an UPB $S=\left\{\left|\psi_{0}>, \cdots,\right| \psi_{m-1}>\right\}$, every $\mid \psi_{i}>(i=0, \cdots, m-1, m+n=N)$ is a product pure-states, such that $S \cup T$ forms a CBUPB of $H$.

(A) According to [1], the number $m$ of states in an UPB must satisfy $m \geq \sum_{i=1}^{M}\left(d_{i}-1\right)+1$, then $n=N-m \leq$ $N-\sum_{i=1}^{M}\left(d_{i}-1\right)-1$.

(B) According to Eq.(1), we have

$$
\sum_{i=0}^{m-1}\left|\psi_{i}><\psi_{i}\right|+\sum_{j=0}^{n-1}\left|\varphi_{j}><\varphi_{j}\right|=I
$$

Here $\left|\psi_{0}>, \cdots,\right| \psi_{m-1}>$ all are products of pure-states, according to the definition of separability of the multipartite systems, the uniform mixture

$$
\bar{\rho}=\frac{1}{N-n}\left\{I-\sum_{j=0}^{n-1}\left|\varphi_{j}><\varphi_{j}\right|\right\}=\sum_{i=0}^{m-1} \frac{1}{m}\left|\psi_{i}><\psi_{i}\right|
$$

is separable.

Sufficiency. Suppose that for a subspace $H_{E S}$ the conditions (A) and (B) both hold. Now, in this $H_{E S}$ we take arbitrarily an orthogonal basis $T=\left\{\left|\varphi_{0}>, \cdots,\right| \varphi_{n-1}>\right\}\left(n \leq N-\sum_{i=1}^{M}\left(d_{i}-1\right)-1\right)$, then according to (B), the density matrix $\bar{\rho}=\frac{1}{N-n}\left\{I-\sum_{j=0}^{n-1}\left|\varphi_{j}><\varphi_{j}\right|\right\}$ is separable, i.e. there must be a decomposition of $\bar{\rho}$ as $\frac{1}{N-n}\left\{I-\sum_{j=0}^{n-1}\left|\varphi_{j}><\varphi_{j}\right|\right\}=\bar{\rho}=\sum_{s} p_{s} \rho_{s}$, where $0<p_{s} \leq 1, \sum_{s} p_{s}=1$, every $\rho_{s}=\left|\Psi_{s}><\Psi_{s}\right|$ is a product state. Then we have

$$
\frac{1}{n} \sum_{j=0}^{n-1}\left|\varphi_{j}><\varphi_{j}\right|=\frac{1}{n}\left\{I-(N-n) \sum_{s} p_{s} \rho_{s}\right\}
$$

Right multiplied two sides of Eq.(4) by $\left|\Psi_{t}\right\rangle$, we obtain 


$$
\sum_{j=0}^{n-1} \lambda_{j, t}\left|\varphi_{j}>=\sum_{s} \mu_{s, t}\right| \Psi_{s}>
$$

where $\lambda_{j, t}=\frac{1}{n}<\varphi_{j} \mid \Psi_{t}>$ and $\mu_{s, t}=\frac{1}{n}\left(\delta_{s t}-(N-n) p_{s}\right)<\Psi_{s} \mid \Psi_{t}>$. But according to the definition of EB and the above supposition, generally Eq.(5) is impossible (the left side is entangled, but the right side is separable), only possibility is that two sides of Eq.(5) both vanish. From $\lambda_{j, t} \equiv 0$, then $\left\langle\varphi_{j} \mid \Psi_{t}\right\rangle=n \lambda_{j, t} \equiv 0$, i.e. all $\mid \Psi_{t}>$ are orthogonal to all $\mid \varphi_{j}>(j=0, \cdots n-1)$. Next, from $\mu_{s, t}=\frac{1}{n}\left(\delta_{s t}-(N-n) p_{s}\right)<\Psi_{s} \mid \Psi_{t}>\equiv 0$ this shows that: When $s \neq t$, then $\left\langle\Psi_{s} \mid \Psi_{t}\right\rangle$ must vanish, i.e. $\left\{\left|\Psi_{s}\right\rangle\right\}$ is a set of orthogonal states; When $s=t$, it leads to $p_{s} \equiv \frac{1}{N-n}$, hence $\sum_{j=0}^{n-1}\left|\varphi_{j}><\varphi_{j}\right|+\sum_{s} \rho_{s}=I$. To compare this with Eqs.(1) and (2), and since $\left\{\mid \Psi_{s}>\right\}$ is a set of orthogonal states, the index $s$ must run over $0,1, \cdots, m-1,(m=N-n)$. Sum up, we obtain a PB $S=\left\{\left|\Psi_{0}>,\right| \Psi_{1}>, \cdots, \mid \Psi_{m-1}>\right\}$ in the orthogonal complementary subspace $H_{T}^{\perp}$. Further, this PB $S$ is an UPB. In fact, if $\Omega$ is an arbitrary product state which is orthogonal to all $\left|\Psi_{i}\right\rangle(i=0, \cdots, m-1) \in S$, then it is in $H_{T}$, because $H=H_{S} \oplus H_{T}$. But this is impossible, since $H_{T}=H_{E S}$ is an ES. This means that $S$, in fact, is an UPB, i.e. $T$ is an EEB and $H_{E S}$ is an EES. According to lemma 1, this $S$ is yet unique.

As for the problem how to decide the separability of a density matrix, we can use some criteria, e.g. the ways in $[16,17]$, etc..

Corollary 1. An EB $T=\left\{\left|\varphi_{0}>, \cdots,\right| \varphi_{n-1}>\right\}\left(n \leq N-\sum_{i=1}^{M}\left(d_{i}-1\right)-1\right)$ is an EEB if and only if the uniform mixture $\bar{\rho}=\frac{1}{N-n}\left\{I-\sum_{j=0}^{n-1}\left|\varphi_{j}><\varphi_{j}\right|\right\}$ is separable.

Proof. According to the definition, the subspace $H_{T}$ spanned by $T$ is an EES if and only if $T$ is an EEB, this means that corollary 1 holds.

By this corollary, if $T=\left\{\left|\varphi_{0}>, \cdots,\right| \varphi_{n-1}>\right\}$ is a EB and the uniform mixture $\bar{\rho}=\frac{1}{N-n}\left\{I-\sum_{j=0}^{n-1}\left|\varphi_{j}><\varphi_{j}\right|\right\}$ is separable, then it determines uniquely an UPB $S=\left\{\left|\psi_{0}>, \cdots,\right| \psi_{m-1}>\right\}$ and a CBUPB $B=S \cup T$, where $\left\{\left|\psi_{0}>, \cdots,\right| \psi_{m-1}>\right\}$ is obtained from the above decomposition $\bar{\rho}=\sum_{s} p_{s} \rho_{s}=\frac{1}{N-n} \sum_{i=0}^{n-1}\left|\psi_{i}><\psi_{i}\right|$ in the proof of Theorem 1.

Corollary 2. If a subspace $H_{E S}$ in $H$ is an EES, then for any orthogonal basis $T=\left\{\left|\varphi_{0}>, \cdots,\right| \varphi_{n-1}>\right\}$ $\left(n \leq N-\sum_{i=1}^{M}\left(d_{i}-1\right)-1\right)$ of $H_{E S}$ the uniform mixture $\tilde{\rho}=\frac{1}{n} \sum_{j=0}^{n-1}\left|\varphi_{j}><\varphi_{j}\right|$ is a bound entangled state[18].

Proof. According to the above Theorem 1, now there is an UPB $S=\left\{\left|\psi_{0}>, \cdots,\right| \psi_{m-1}>\right\}(m=N-n)$ and $\tilde{\rho}=\frac{1}{N-m}\left\{I-\sum_{i=0}^{m-1}\left|\psi_{i}><\psi_{i}\right|\right\}$, then this corollary is just a known theorem (the Theorem 1) in [1,3].

We notice that a $\left(N-\sum_{i=1}^{M-1}\left(d_{i}-1\right)-1\right)$-dimensional EEB (or EES) is unextendible, i.e. there is no other EEB (or EES) which contains this EEB (or EES) as a proper subset (or a proper subspace). In fact, according to [1], in this case the dimensionality of the UPB is minimum, so the dimensionality $\left(N-\sum_{i=1}^{M-1}\left(d_{i}-1\right)-1\right)$ of EEB (or EES) is maximal.

Now we consider the problem what is the general structures of orthogonal complete bases. Generally, in an orthogonal complete basis there may be some entangled pure-states, especially, these entangled pure-states can form an EB, even an EEB. All possible cases can be discussed as follows.

(I) In $B$ there is no EB. Now, a general orthogonal complete basis of $H$ is in form as $B=S \cup R=$ $\left\{\left|\psi_{0}>, \cdots,\right| \psi_{m-1}>,\left|\Theta_{0}>, \cdots,\right| \Theta_{N-m-1}>\right\}$, where $S=\left\{\left|\psi_{0}>, \cdots,\right| \psi_{m-1}>\right\}$ is an orthogonal product basis, and $R=\left\{\left|\Theta_{0}>, \cdots,\right| \Theta_{N-m-1}>\right\}$ is a set of orthogonal entangled pure-states, but $R$ is not an EB, i.e. there may be a linear combination $\sum_{i=0}^{N-m-1} c_{i} \mid \Theta_{i}>$ which is separable. This means that if $m<N, S$ must be an extendible product basis. Here there are two extreme cases: (i) $n=N$, then $B$ is a complete orthogonal product basis, e.g. the common standard basis $\left\{\left|j_{\alpha_{1}}>\otimes \cdots \otimes\right| j_{\alpha_{M}}>\right\}\left(j_{\alpha_{i}}=0,1, \cdots, d_{i}-1\right.$ for $\left.i=1, \cdots, M\right)$ is such a basis. (ii) $n=0$, then all pure-states in $B$ are entangled, e.g. the known Bell's basis in the bipartite qubit systems is such a basis (notice that it is not an $\mathrm{EB}$ ).

(II) In $B$ there is an EB $T=\left\{\left|\varphi_{0}>, \cdots,\right| \varphi_{n-1}>\right\}$. Here, there are only two possibilities: (i) The mixed-state $\bar{\rho}=$ 
$\frac{1}{N-n}\left\{I-\sum_{j=0}^{n-1}\left|\varphi_{j}><\varphi_{j}\right|\right\}$ is separable, then according to the above theorem 1, $T$ just is an EEB. This means that there must be an UPB $S=\left\{\left|\psi_{0}>, \cdots,\right| \psi_{N-n-1}>\right\}$, and $B=S \cup T=\left\{\left|\psi_{0}>, \cdots,\right| \psi_{N-n-1}>,\left|\varphi_{0}>, \cdots,\right| \varphi_{n-1}>\right\}$ is an CBUPB. (ii) The mixed-state $\bar{\rho}=\frac{1}{N-n}\left\{I-\sum_{j=0}^{n-1}\left|\varphi_{j}><\varphi_{j}\right|\right\}$ is entangled. In order to discuss this case, we need to use the following lemma:

Lemma 2. If $T=\left\{\left|\varphi_{0}>, \cdots,\right| \varphi_{n-1}>\right\}$ is an $E B$ and the mixed-state $\bar{\rho}=\frac{1}{N-n}\left\{I-\sum_{j=0}^{n-1}\left|\varphi_{j}><\varphi_{j}\right|\right\}$ is entangled, then in the orthogonal complementary space $H_{T}^{\perp}$ there is no $(N-n)$-dimensional orthogonal product basis.

Proof. Conversely, suppose that in $H_{T}^{\perp}$ there is an orthogonal product basis $S=\left\{\left|\psi_{0}>, \cdots,\right| \psi_{N-n-1}>\right\}$, then $S$ must be an UPB, since $T$ is an EB. According to the Theorem 1, in this case $\bar{\rho}=\frac{1}{N-n}\left\{I-\sum_{j=0}^{n-1}\left|\varphi_{j}><\varphi_{j}\right|\right\}$ must be separable, but this contradicts to the supposition.

By using of Lemma 2, we know that in the case (ii) the form of an orthogonal basis of $H_{T}^{\perp}$ must be as $\left\{\left|\psi_{0}>, \cdots,\right| \psi_{r-1}>,\left|\phi_{0}>, \cdots,\right| \phi_{s-1}>\right\}(1 \leq r \leq N-n-2,0 \leq s \leq N-n-1, r+s=N-n-1)$, where $S=$ $\left\{\left|\psi_{0}>, \cdots,\right| \psi_{r-1}>\right\}$ is a $\mathrm{PB}$, and $T^{\prime}=\left\{\left|\phi_{0}>, \cdots,\right| \phi_{s-1}>\right\}$ is a set of orthogonal entangled pure-states, but it is not an EB (i.e. some linear combination of $\left|\phi_{0}>, \cdots,\right| \phi_{n-1}>$ may be a separable state). Now the basis of $H$ is in form as $B=S \cup T^{\prime} \cup T=\left\{\left|\psi_{0}>, \cdots,\right| \psi_{r-1}>,\left|\phi_{0}>, \cdots,\right| \phi_{s-1}>,\left|\varphi_{0}>, \cdots,\right| \varphi_{n-1}>\right\}$. Here we notice that $T^{\prime} \cup T=\left\{\left|\phi_{0}>, \cdots,\right| \phi_{s-1}>,\left|\varphi_{0}>, \cdots,\right| \varphi_{n-1}>\right\}$ and the basis $R$ in the case (I) are very much alike, however in the former there is an EB in $B$.

Sum up, the forms of a general orthogonal complete bases $B$ can be tabulated as follows:
The first kind: In $B$ there is no EB -
(Special: $\mathbf{0} \mathbf{0} \|$, and $\|----)$
$\square \square \square \square \| \equiv \equiv \equiv \equiv$
The second kind: In $B$ there is an EB - (i) $\bar{\rho}$ is separable-
- (ii) $\bar{\rho}$ is entangled-
घ[1] $\mid----===$
(it is a CBUPB by a proper choice of $S$ )

where denotes a PB; - - - - denotes the basis consisting of entangled pure-states, but it is not an EB; denotes an $\mathrm{UPB} ;===$ denotes an $\mathrm{EB}$, but it is not an $\mathrm{EEB} ;====$ denotes an $\mathrm{EEB}$. The symbol $\|$ denotes the partition between two parts $\mathrm{PB}$ and $\mathrm{EB}$.

Applications and examples in quantum mechanics and quantum information

1. A CBUPB constructed from the GenTiles1. About the requirements and applications of the PBs and UPBs in the quantum information, see [2,3]. A known UPB is the GenTiles given by DiVincenzo, Terhal et al.[2,3]. By using of the symbols as in [2,3], in $H=H_{n} \otimes H_{n}(n$ is even and $n \geq 4)$ the GenTiles1 $\left\{\left|\mathbf{V}_{m k}>,\right| \mathbf{H}_{m k}>, \mid \mathbf{F}>\right\}$ is defined by (here we have taken their normalization).

$$
\begin{aligned}
& \left|\mathbf{V}_{m k}>=\frac{2}{n}\right| k>\otimes\left|\omega_{m, k+1}>=\frac{2}{n}\right| k>\otimes \sum_{j=0}^{\frac{n}{2}-1} \omega^{j m} \mid j+k+1 \bmod n>, \omega=e^{\frac{i 4 \pi}{n}} \\
& \left|\mathbf{H}_{m k}>=\frac{2}{n}\right| \omega_{m, k}>\otimes|k>,| \mathbf{F}>=\frac{1}{n^{2}} \sum_{i=0}^{n-1} \sum_{j=0}^{n-1}|i>\otimes| j>
\end{aligned}
$$

where $m=1, \cdots, \frac{n}{2}-1, \quad k=0, \cdots, n-1$.If we take $\left|\mathbf{F}_{r}>=\right| r>\otimes \mid r>, \quad(r=0,1, \cdots, 2(n-1))$, then $\left\{\left|\mathbf{V}_{m k}>,\right| \mathbf{H}_{m k}>,|\mathbf{F}>,| \mathbf{F}_{r}>\right\}$ (the total of states is $n^{2}$ ) is a linearly independent group of normalized states. Now, we define the normalized states $\mid \varphi_{r}>(r=0, \cdots, 2(n-1))$ by induction as follows (i.e. the Schmidt's orthogonalization)

$$
\begin{aligned}
& \mid \varphi_{0}>=\eta_{0}\left\{\left|\mathbf{F}_{0}>-\sum_{m=1}^{\frac{n}{2}-1} \sum_{k=0}^{n-1}\left(<\mathbf{V}_{m k}\left|\mathbf{F}_{0}>\right| \mathbf{V}_{m k}>+<\mathbf{H}_{m k}\left|\mathbf{F}_{0}>\right| \mathbf{H}_{m k}>\right)-<\mathbf{F}\right| \mathbf{F}_{0}>\mid \mathbf{F}>\right\} \\
& =\eta_{0}\left(|0>\otimes| 0>-\frac{2}{n} \mathbf{H}_{00}-\frac{1}{n^{2}} \mid \mathbf{F}>\right), \eta_{0}=\frac{4 n^{8}}{4\left(n^{4}-2 n^{2}-1\right)^{2}+(n-2)^{2}\left(4 n^{2}+1\right)^{2}+4 n^{2}(n-1)^{2}+n^{2}}
\end{aligned}
$$




$$
\begin{aligned}
\varphi_{r} & =\eta_{r}\left\{\left|\mathbf{F}_{r}>-\sum_{m=1}^{\frac{n}{2}-1} \sum_{k=0}^{n-1}\left(<\mathbf{V}_{m k}\left|\mathbf{F}_{r}>\right| \mathbf{V}_{m k}>+<\mathbf{H}_{m k}\left|\mathbf{F}_{r}>\right| \mathbf{H}_{m k}>\right)-<\mathbf{F}\right| \mathbf{F}_{r}>\left|\mathbf{F}>-\sum_{s=0}^{r-1}<\varphi_{s}\right| \mathbf{F}_{r}>\mid \varphi_{s}>\right\} \\
& =\eta_{r}\left\{\left|\mathbf{F}_{r}>-\frac{2}{n} \mathbf{H}_{r r}-\left(1+\sum_{s=0}^{r-1} \eta_{s}\right) \frac{1}{n^{2}}\right| \mathbf{F}>\right\}, r=1, \cdots, 2(n-1)
\end{aligned}
$$

where $\eta_{r}(r=0, \cdots, 2(n-1))$ are normalization factors, which are also determined by induction. Therefore the set $\left\{\left|\mathbf{V}_{m k}>,\right| \mathbf{H}_{m k}>,|\mathbf{F}>,| \varphi_{r}>\right\}\left(m=1, \cdots, \frac{n}{2}-1, k=0, \cdots, n-1, r=0, \cdots, 2 n-1\right)$ forms an orthogonal complete basis of $H=H_{n} \otimes H_{n}$. Since the GenTiles1 $\left\{\left|\mathbf{V}_{m k}>,\right| \mathbf{H}_{m k}>, \mid \mathbf{F}>\right\}$ is an UPB[2,3] and $\left\{\mid \varphi_{r}>\right\}$ is in $H_{\text {GenTiles1 }}^{\perp}\left(H_{\text {GenTiles1 }}\right.$ is the subspace spanned by the GenTiles1), every $\mid \varphi_{r}>$ is entangled, the set $\left\{\mid \varphi_{r}>\right\}$ is an EEB, and $\left\{|\mathbf{V} m k>,| \mathbf{H}_{m k}>,|\mathbf{F}>,| \varphi_{r}>\right\}$ is a CBUPB . In addition, according to the Theorem 1 and Corollary $2, \bar{\rho}=\frac{1}{(n-1)^{2}}\left\{I-\sum_{r=0}^{2(n-1)}\left|\varphi_{r}><\varphi_{r}\right|\right\}$ is separable, and $\tilde{\rho}=\frac{1}{2 n-1} \sum_{r=0}^{2(n-1)}\left|\varphi_{r}><\varphi_{r}\right|$ is a bound entangled state.

This example clearly shows the use of the GenTiles1 and the theorem 1. A similar result can be obtained from the GenTiles2[2,3].

2. Some bound entangled states. In the quantum information, one need to study the problem what the entanglement can be distilled, because this relates the quantum communication problems. A bound entangled state is such a entangled state that no entanglement can be distilled[18]. If in the system there is an $\operatorname{EEB} T=\left\{\left|\varphi_{0}>, \cdots,\right| \varphi_{n-1}>\right\}$, then according to the above Corollary 2, the uniform mixture $\tilde{\rho}=\frac{1}{n} \sum_{j=0}^{n-1}\left|\varphi_{j}><\varphi_{j}\right|$ is entangled and is a bound entangled state, this is a standard case as in $[1,3]$. However, we find that there may be yet other bound entangled states with similar forms as follows. For instance, suppose that in the system there is a $\operatorname{EB} T=\left\{\left|\varphi_{0}>, \cdots,\right| \varphi_{n-1}>\right\}$, but the mixed-state $\bar{\rho}=\frac{1}{N-n}\left\{I-\sum_{j=0}^{n-1}\left|\varphi_{j}><\varphi_{j}\right|\right\}$ is entangled, then according to the above classification tab, there are a PB $S=\left\{\left|\psi_{0}>, \cdots,\right| \psi_{r-1}>\right\}$ and a $T^{\prime}=\left\{\left|\phi_{0}>, \cdots,\right| \phi_{s-1}>\right\}(r+s+n=N)$, which is a set of orthogonal entangled pure-states but not an EB, they form together an orthogonal complete basis $B=S \cup T^{\prime} \cup T$. Let

$$
\widetilde{\rho^{\prime}}=\frac{1}{s+n}\left\{\sum_{i=0}^{s-1}\left|\phi_{k}><\phi_{k}\right|+\sum_{j=0}^{n-1}\left|\varphi_{j}><\varphi_{j}\right|\right\}=\frac{1}{N-r}\left\{I-\sum_{k=0}^{r-1}\left|\psi_{k}><\psi_{k}\right|\right\}
$$

Since $\widetilde{\rho^{\prime}}$ contains the component part constructed by the states in $T^{\prime}$, we cannot affirm that $\widetilde{\rho^{\prime}}$ must be a entangle state for any $T^{\prime}$. However in many cases, by a proper choice of $T^{\prime}, \widetilde{\rho}^{\prime}$ always can become entangled. Notice that the related argument in the proof about $\tilde{\rho}$ to be a bound entangled state (see the proof of Theorem 1 in $[1,3]$ ), obviously, is still completely effective for $\widetilde{\rho^{\prime}}$, i.e. in present case also no entanglement can be distilled. Therefore $\widetilde{\rho}^{\prime}$ is a bound entangled state. This example shows the multiformity of bound entangled states with the uniform mixture forms.

At last, we consider the problem of transformations. Since at present we only discuss the orthogonal bases, we cannot use the general local operations and classical communication[13]. However, we can use the local unitary transformations.

Theorem 2. The structure form of a general orthogonal complete basis $B=S \cup T=\left\{\left|\psi_{0}>, \cdots,\right| \psi_{n-1}>,\left|\varphi_{0}>, \cdots,\right| \varphi_{k-1}>\right\}(n+k=N)$ is invariant under a local operation as

$$
\begin{aligned}
& G: B \longrightarrow B^{\prime}=S^{\prime} \cup T^{\prime}=\left\{\left|\psi_{0}^{\prime}>, \cdots,\right| \psi_{n-1}^{\prime}>,\left|\varphi_{0}^{\prime}>, \cdots,\right| \varphi_{k-1}^{\prime}>\right\} \\
& \quad\left|\psi_{i}^{\prime}>=u\left(d_{1}\right) \otimes u\left(d_{2}\right) \otimes \cdots \otimes u\left(d_{M}\right)\left(\mid \psi_{i}>\right),\right| \varphi_{j}^{\prime}>=u\left(d_{1}\right) \otimes u\left(d_{2}\right) \otimes \cdots \otimes u\left(d_{M}\right)\left(\mid \varphi_{j}>\right)
\end{aligned}
$$

where $u\left(d_{i}\right)\left((i=1, \cdots, M)\right.$ are arbitrary $d_{i} \times d_{i}$ unitary matrixes.

Proof. Obviously, an interior product of any two states is invariant under this $G$, then an orthogonal complete basis will be changed into another orthogonal complete basis under $G$, i.e. $G$ indeed is a transformation of general orthogonal complete bases. Next, for a pure-state $\mid \psi>$, the pure-state $\left|\psi^{\prime}\right\rangle=u\left(d_{1}\right) \otimes u\left(d_{2}\right) \otimes \cdots \otimes u\left(d_{M}\right)(\mid \psi>)$ is a product state, if and only if $|\psi\rangle$ is a product state itself. This means that if in $B$ there is no EB, then in $B^{\prime}=G(B)$ there is yet no EB. In addition, when in $B$ there is an EB $T$, the corresponds uniform mixture is $\bar{\rho}$, then the uniform mixture $\bar{\rho}^{\prime}=u\left(d_{1}\right) \otimes u\left(d_{2}\right) \otimes \cdots \otimes u\left(d_{M}\right) \bar{\rho} u^{-1}\left(d_{M}\right) \otimes u^{-1}\left(d_{M-1}\right) \otimes \cdots \otimes u^{-1}\left(d_{1}\right)$ is separable, if and only if $\bar{\rho}$ is separable itself. According to the above classification tab and these discussions, it has been proved that the structure form of a general orthogonal complete basis $B$ is invariant under $G$. 
This theorem can be used to create various orthogonal complete bases (in the same type) from given one.

[1] C. H. Bennett, D. P. DiVincenzo, T. Mory, P. W. Shorz, J. A. Smolin, and B. M. Terhalx, Phys. Rev. Lett., 82(1999)5385.

[2] D. P. DiVincenzo and B. M. Terhal, quant-ph/0008055.

[3] D. P. DiVincenzo , T. Mory, P. W. Shorz, J. A. Smolin and B. M. Terhalx, Comm. Math. Phys., $238(2003) 379$.

[4] B. M. Terhal, Lin. Alg. Appl., 323(2000)61.

[5] B. M. Terhal, J. Theor. Comp. Sci., 287(2002)313.

[6] A. Acin, D. Bru $\beta$, M. Lewenstein, and A. Sanpera, Phys. Rev. Lett., 87(2001)040401.

[7] S. Chaturvedi, quant-ph/0105125.

[8] A. O. Pittenger and M. H. Rubin, quant-ph/0207024.

[9] A. O. Pittenger, Linear Algebr. Appl. 359(2003)235.

[10] S. D. Rinaldis, quant-ph/0304027.

[11] S. Bravy, quant-ph/0310172.

[12] C. Altafini, quant-ph/0405124.

[13] C. H. Bennett, D. P. DiVincenzo, J. Smolin, and W. K. Wootters, Phys. Rev. A, 54(1996)3824.

[14] W. Dür, G. Vidal and J. I. Cirac, Phys. Rev. A, 62(2000)062314.

[15] L. H. Kauffmana and S. J. Lomonaco Jr., quant-ph/0304091.

[16] S. Wu, X. Chen and Y. Zhang, Phys Lett. A, 275(2000)244.

[17] K, Chen and L. A. Wu, Phys. Lett. A, 306(2002)14.

[18] M. Horodecki, P. Horodecki, and R. Horodecki, Phys. Rev. Lett., 80(1998)5239. 